\title{
High-sensitivity and large-dynamic-range refractive index sensors employing weak composite Fabry-Perot cavities
}

\author{
Pengcheng Chen ${ }^{1}$, Xuewen Shu ${ }^{1, *}$, Hanyuan CaO ${ }^{1}$, Kate Sugden ${ }^{2}$ \\ ${ }^{1}$ Wuhan National Laboratory for Optoelectronics \& School of Optoelectronic Science and Engineering, Huazhong University of Science and \\ Technology, Wuhan 430074, China \\ ${ }^{2}$ Aston Institute of Photonic Technologies, Aston University, Birmingham B4 7ET, UK \\ * Corresponding author: xshu@hust.edu.cn
}

Received XX Month XXXX; revised XX Month, XXXX; accepted XX Month XXXX; posted XX Month XXXX (Doc. ID XXXXX); published XX Month XXXX

\begin{abstract}
Most sensors face a common tradeoff between high sensitivity and large dynamic range. We demonstrate here an all-fiber refractometer based on a dual cavity Fabry-Perot interferometer (FPI), which possesses the advantage of both high sensitivity and large dynamic range. Since the two composite cavities have a large cavity length difference, one can observe both fine and coarse fringes, which correspond to the long cavity and short cavity, respectively. The short cavity FPI and the use of an intensity demodulation method, mean that the individual fine fringe dips correspond to a series of quasi-continuous highly sensitive zones for refractive index measurement. By calculating the parameters of the composite FPI, we find that the range of the ultrasensitive zones can be considerably adjusted to suite the end requirements. The experimental trends are in good agreement with the theoretical predictions. The coexistence of high sensitivity and large dynamic range in a composite FPI is of great significance to practical RI measurements. (c) 2017 Optical Society of America
\end{abstract}

OCIS codes: (060.2370) Fiber optics sensors; (230.4000) Microstructure fabrication; (120.3180) Interferometry; (120.2230) Fabry-Perot. (230.1150) All-optical devices.

http://dx.doi.org/10.1364/OL.99.099999

Sensors with both high sensitivity and large dynamic range are very desirable for many applications, however, most sensors face a tradeoff between the two. Refractive index (RI) sensors, which are important sensors in the fields of physics, chemistry, biomedicine and environmental science, also have such a dilemma. Most effort to date has been to improve the sensitivity of such sensors. However, for wavelength demodulated sensors such as micro/nanofiber devices [1-7], long-period fiber gratings [8-9], etc., their high RI sensitivities usually only apply to a very small RI range. Beyond the specified RI range, the sensing performance will be greatly reduced or even stop functioning. Intensity demodulated sensors include devices such as: microfibers [10]; through fiber core micro channels [11-13]; Michelson interferometers (MI) [14]; multimode interferometers (MMI) [15], and Fabry-Perot interferometers (FPIs) [16-19]. The sensitivities of those reported intensity demodulated schemes based on microfibers, micro channels and FPIs are relatively low. Although the sensitivities of the MI and MMI based schemes are higher they still only work over for high sensitivity over a small RI range. Recently, we also demonstrated a simple and robust intensity demodulated RI sensor [20], which is based on a weak compositecavity Fabry-Perot interferometer that was created using a femtosecond laser (fs-laser). Using an intensity tracking interrogation method to monitor one interference fringe dip strength, a high resolution up to $4.0 \times 10^{-7}$ was achieved in a small range around the discovered sensitive zone.

In this paper, we report that such a composite-cavity FPI has the potential to solve the tradeoff difficulty of high sensitivity and large dynamic range. Our further investigation if the properties of these devices shows that different interference fringe dips of the composite FPI can correspond to a series of different ultrasensitive zones for RI measurement. Therefore, high sensitivity and large dynamic range can co-exist in such structures. We find that the range of the ultra-sensitive zones can be significantly adjusted and tailored by adjusting the parameters of the composite-cavity FPI. The experimental trends shown are in good accord with the theoretical analysis and simulations.

Figure 1 shows the schematic diagram of the proposed composite FPI, which comprises of a well-cleaved SMF and a small refractive-index-modified-dot (RIMD) in the fiber core. Due to the refractive index difference, the composite cavity has three 
reflectors: SMF core/RIMD (Mirror1), RIMD/SMF core (Mirror2) and SMF core/surrounding medium (Mirror3), respectively.

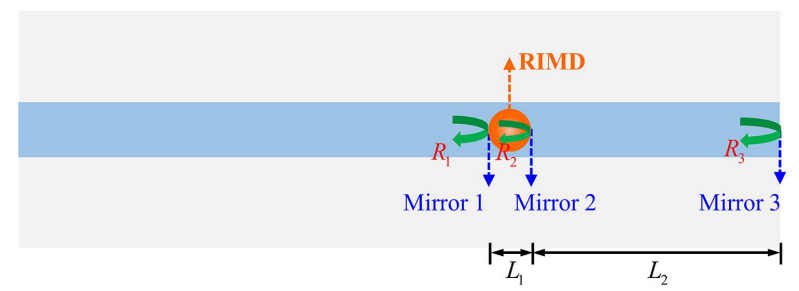

Fig. 1Schematic diagram of the proposed composite FPI.

The light propagating in the fiber core is reflected by the three mirrors and generates a three-wave FPI pattern in fiber. The interference pattern consists of both broad fringes and fine fringes that result from the interference of light inside the composite cavity formed by Mirrors 1, 2 and 3. For $n_{S R I}<n_{c o}\left(n_{S R I}\right.$ is the RI of the surrounding medium), the normalized strength of the interference fringes can be modeled using the following threebeam optical interference equation [20]:

$$
\begin{aligned}
I= & R_{1}+A^{2} R_{2}+B^{2} R_{3}-2 \sqrt{R_{1} R_{2}} A \cos \left(2 \varphi_{1}\right)- \\
& 2 \sqrt{R_{1} R_{3}} B \cos \left(2 \varphi_{1}+2 \varphi_{2}\right)+2 A B \sqrt{R_{2} R_{3}} \cos \left(2 \varphi_{2}\right)
\end{aligned}
$$

Where $A=\left(1-\alpha_{1}\right)\left(1-\gamma_{1}\right)\left(1-R_{1}\right), B=\left(1-\alpha_{1}\right)\left(1-\gamma_{1}\right)\left(1-R_{1}\right)(1$ $\left.-\alpha_{2}\right)\left(1-\gamma_{2}\right)\left(1-R_{2}\right) ; \varphi_{j}=4 \pi n_{j} L_{j} / \lambda$ is the phase shift in the cavity $j(j=1,2) ; n_{j}$ and $L_{j}$ are the refractive index and length of the cavity $j$, respectively; $\alpha_{1}, \alpha_{2}$ are the intensity attenuation factors of the Mirror 1 and Mirror 2, respectively; $\gamma_{1}, \gamma_{2}$ are defined as the transmission loss factors of the Cavity 1 and Cavity 2 , respectively. $R_{i}$ is the intensity reflectivity of the three reflectors, $i(i=1,2,3)$, which can be calculated by the Fresnel formula, $R_{1}=R_{2}=\left[\Delta n /\left(n_{d o t}+n_{c o}\right)\right]^{2}, R_{3}=\left[\left(n_{c o}-n_{S R I}\right) /\left(n_{c o}+\right.\right.$ $\left.\left.n_{S R I}\right)\right]^{2}$, here $n_{c o}$ is the core RI of SMF, $n_{d o t}$ is the RI of the RIMD and $\Delta n=n_{d o t}-n_{c o}$. Using Eq. (1), we can calculate the normalized reflection spectra from $1300 \mathrm{~nm}$ to $1700 \mathrm{~nm}$ with parameters $\alpha_{1}=\alpha_{2}=0.3, \gamma_{1}=\gamma_{2}=0.2, \Delta n=-0.018, n_{c o}=1.46, L_{1}=4 \mu \mathrm{m}, L_{2}=$ $50 \mu \mathrm{m}$, respectively. The front and top view of calculated reflection spectra under various surrounding RIs are shown in Fig. 2(a) and (b), respectively. From which, one can clearly see that the reflection spectrum features dense fringes are modulated by a lowfrequency envelope. The free spectral range (FSR) of these dense fringes corresponds to the $50 \mu \mathrm{m}$ long cavity 2 , while the lowfrequency envelope stems from the $4 \mu \mathrm{m}$ cavity 1 .

Interestingly, one can clearly see that the different interference fringe dips correspond to different ultra-sensitive zones for RI measurement (shown in blue/green on the figures). However, these ultra-sensitive zones are discrete, which is not convenient for continuous RI measurement. To reduce the spacing of these discrete ultra-sensitive zones, one can increase the length of cavity $2\left(L_{2}\right)$, by changing the distance between the short cavity and the end of the SMF. The calculated reflection spectrum with
$L_{2}=500 \mu \mathrm{m}$ is shown in Fig. 2(c), where the longer $L_{2}$ increase the number of peaks so that they become quasi continuous. For comparison, we also calculated the reflection spectrum with $L_{1}=0$ (i.e., the device is not a composite cavity but a single cavity), as shown in Fig. 2(d).
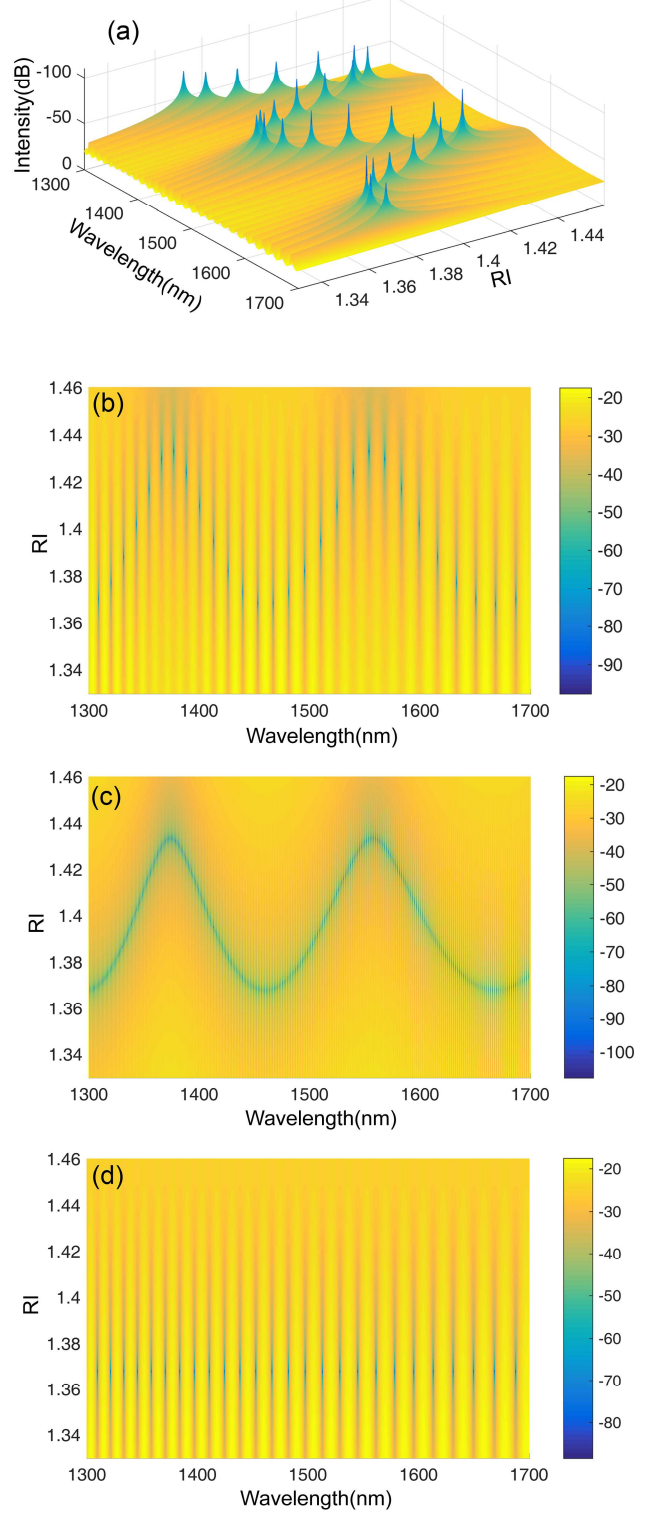

Fig. 2(a) the front and (b) top view of the composite FPI $\left(L_{1}=4 \mu \mathrm{m}\right.$, $L_{2}=50 \mu \mathrm{m}$ ) simulation reflection spectrum for RI change, respectively. (c) The top view of the composite FPI ( $\left.L_{1}=4 \mu \mathrm{m}, L_{2}=500 \mu \mathrm{m}\right)$ simulation reflection spectrum for RI change. (d) The top view of the single FPI $\left(L_{1}=0, L_{2}=50 \mu \mathrm{m}\right)$ simulation reflection spectrum for RI change.

Compared Fig. 2(d) with (b), one can see that, without the envelope modulation caused by the short cavity, the ultra-sensitive 
regions of the respective interference dips point to the same RI value (at 1.37), which means the ultra-sensitive zone is limited to a small range of measurable RI values as you travel from the minimum to maximum of the peaks. While for the composite FPI ( $L_{1}=4 \mu \mathrm{m}, L_{2}=500 \mu \mathrm{m}$ ), the ultra-sensitive zone can be widened to cover the range of $\sim 1.37$ to $\sim 1.43$.

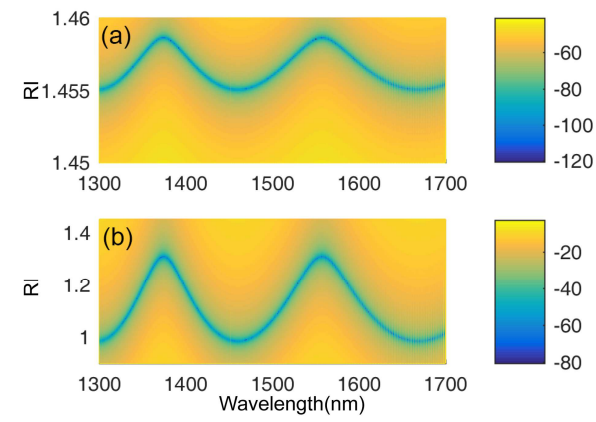

Fig. 3 Calculated reflection spectra for (a) $\Delta n=-0.001$ and (b) -0.11 .
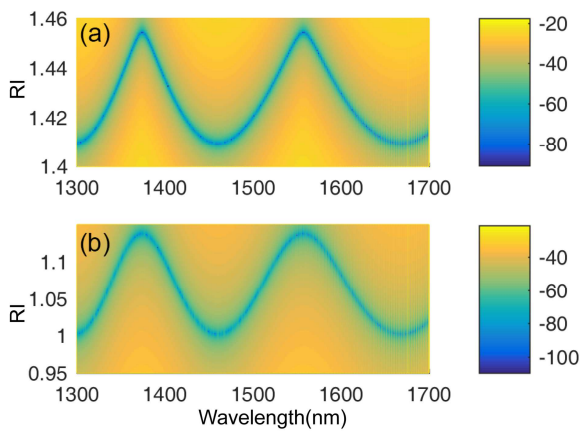

Fig. 4 Calculated reflection spectra for (a) $\alpha_{1}=\alpha_{2}=0.01$ and (b) 0.75 .

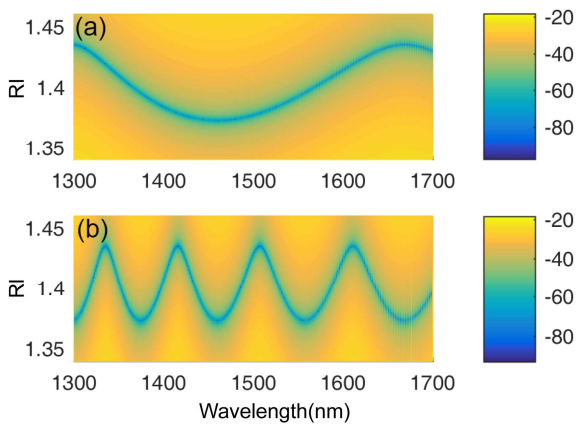

Fig. 5 Calculated reflection spectra for (a) $L_{1}=2 \mu \mathrm{m}$ and (b) $8 \mu \mathrm{m}$.

To further investigate how the range of the ultra-sensitive zones changes with different cavity parameters, we simulated the same composite cavity FPI as Fig.2(c) whilst changing a certain parameter each time. Fig. 3(a)-(b) show the spectra for $\Delta n=-$ 0.001 and -0.11 , respectively. Compared with Fig. 2(c) [ $\Delta n=-$ $0.018]$, one can see that the range of the ultra-sensitive zone is greatly influenced by the value of $\Delta n$. The ranges of the ultrasensitive RI zones are located at 1.455-1.458, 1.37-1.43 and 1.0-1.3, for $\Delta n=-0.001,-0.018$ and -0.11 respectively. As $\Delta n$ decreases, the ultra-sensitive zones shift to a smaller RI range and the range of the ultra-sensitive zone widens. The greater the RI modulation $(\Delta n)$, the smaller and wider the ultra-sensitive RI range. Therefore, the RI measurement from gas to liquid can be realized by selecting reasonable design parameters. To a degree, it also means that the $\Delta n$ parameter is more favorable for low RI liquid or even gas measurements. Fig. 4(a),(b) show the spectra for the loss factor $\alpha_{1}=\alpha_{2}=0.01$ and 0.75 , respectively. Compared with Fig. 2(c) $\left[\alpha_{1}=\alpha_{2}=0.3\right]$, one can see that the range of the ultra-sensitive zone is also greatly influenced by the value of the loss factor. The ultra-sensitive RI zones are located at 1.410-1.455, 1.37-1.43 and 1.00-1.15 for $\alpha_{1}=\alpha_{2}=0.01,0.3$ and 0.75 , respectively. Increasing the loss factor, results in specific performance trends that are similar to those with the decrease in $\Delta n$. Fig. 5 (a), (b) show the spectra for an axial RIMD length $L_{1}=2$ and $8 \mu \mathrm{m}$, respectively. Compared with Fig. 2(c) [ $\left.L_{1}=4 \mu \mathrm{m}\right]$, one can see that the value of $L_{1}$ is inversely proportional to the envelope period, but almost has no influence on the range of the ultra-sensitive zones (i.e. 1.371.43).
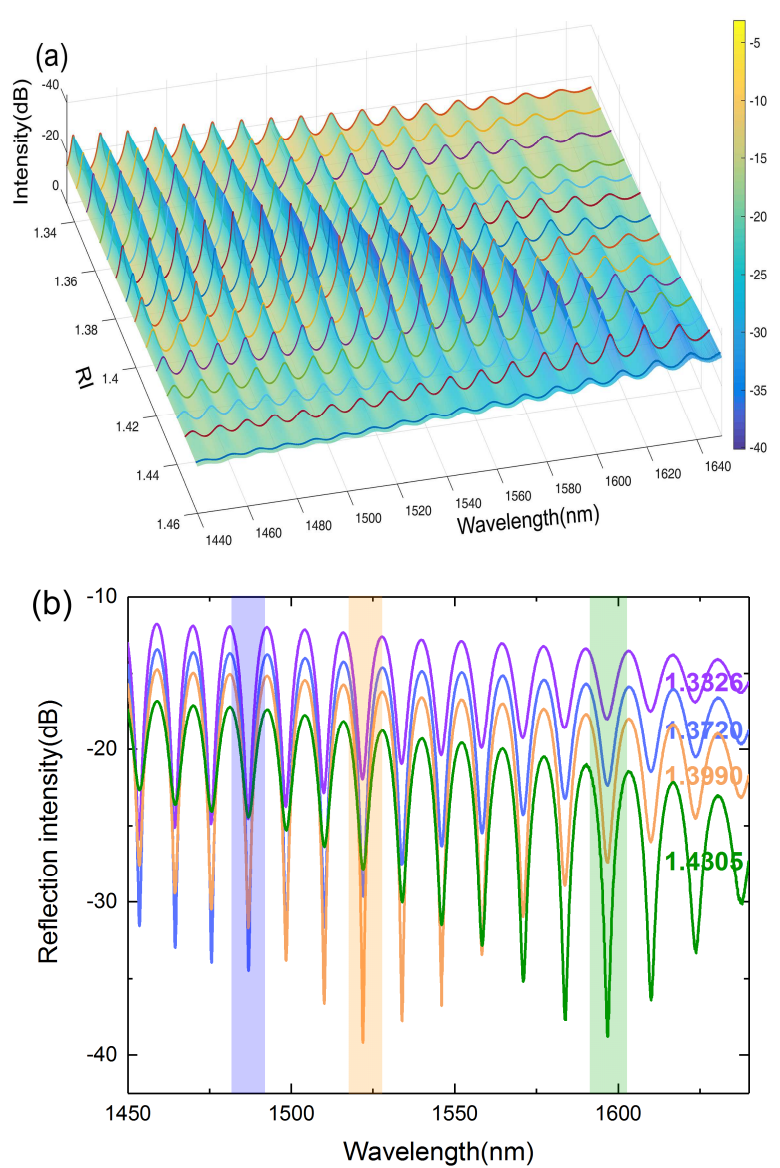

Fig. 6 The front view (a) of and typical (b) measured reflective spectra of the composite FPI in different surrounding RI. 


\begin{tabular}{llllllll}
-40 & -35 & -30 & -25 & -20 & -15 & -10 & -5 \\
\hline & & & & & & &
\end{tabular}

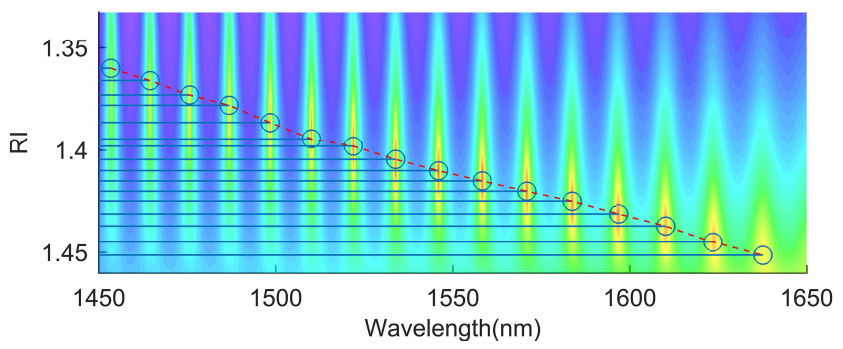

Fig. 7 Top view of the measured spectra versus surrounding RI.

A simple structure was fabricated to verify the above theoretical model. An RIMD was induced into an end well cleaved SMF using a fs-laser. The fabrication of this device is very straightforward and the specific fabrication equipment and process are similar to our previous work [20].

We investigated the response of the composite FPI to surrounding RI using sucrose solutions with different concentrations. Fig. 6(a) shows the front view of the experimental reflection spectrum of a sample with designed $L_{2}=70 \mu \mathrm{m}$ under different RI. It can be clearly observed that different interference fringes reach the ultra-sensitive zones at different RIs, the trend of which are consistent with the theory calculations. To observe the spectral evolution more thoroughly, Fig. 6(b) shows the reflected spectrum under different RI conditions. The FSR of the fine interference fringes is $\sim 11.6 \mathrm{~nm}$, and thus the corresponding cavity length can be calculated by $L_{2}=\lambda_{1} \lambda_{2} / 2 n_{c o} F S R=66.8 \mu \mathrm{m}$ (at $1500 \mathrm{~nm}$ and the RI of the core $n_{c o}=1.46$ ), which is very close to the designed value. It indicates that $L_{2}$ can be controlled easily. The coarse fringes results from the short dot-cavity.

Fig. 7 shows the top view of the measured spectra versus surrounding RI. The interference fringe dip at the wavelength of $1453.4 \mathrm{~nm}$ reaches the ultra-sensitive zone at the RI of $\sim 1.359$, while the interference fringe dip at the wavelength of $1637.68 \mathrm{~nm}$ reaches the ultra-sensitive zone at the RI of $\sim 1.452$, as shown in Fig.6(a) and Fig. 7. With the increase in wavelength of the deepest interference fringe dip at different surrounding RIs successive ultra-sensitive zones are reached. These results indicate that our composite FPI device can achieve the co-existence of high sensitivity and larger dynamic range in RI measurement, the trends of which are in good accord with the theoretical predictions. Moreover, the red dash curve in Fig. 7 shows the recorded deepest fringe wavelength location versus RI. One can see that the location of the deepest interference fringe has an obvious "red shift" in the RI range of 1.359 to 1.452 , which is also in good accord with the theoretical calculation. Potentially, one may also use this location information as an additional indicator to decode the RI information.

In conclusions, we discover the co-existence of high sensitivity and large dynamic range in a composite intrinsic FPI for RI sensing. To verify such a characteristic, a simple configuration is proposed, which is constituted by simply inducing a refractive-indexmodified-dot (RIMD) in a fiber core using a fs-laser. The short
RIMD cavity defines the large dynamic range, while the long SMF core cavity provides the high sensitivity ability for RI sensing. To the authors' knowledge, it is the first time that this important phenomenon in a composite FPI for RI measurement is observed. By calculating the parameters of the composite FPI, we find that the range of the ultra-sensitive zones can be significantly adjusted. Additional merits, such as low cost components, a simple and compact structure, good robustness, and the ability to overcome the temperature / RI cross-sensitivity [20] make this device a very promising candidate for practical sensing applications.

Funding. Director Fund of WNLO; National 1000 Young Talents Program, China; 111 Project (No. B07038)

\section{References}

1. J. Li, L. P. Sun, S. Gao, Z. Quan, Y. L. Chang, Y. Ran, L. Jin, and B. O. Guan, Opt. Lett. 36, 3593 (2011).

2. L. P. Sun, J. Li, S. Gao, L. Jin, Y. Ran, and B. O. Guan, Opt. Lett. 39, 3531 (2014).

3. X. Fang, C. R. Liao, and D. N. Wang, Opt. Lett. 35, 1007 (2010).

4. S. Gao, W. Zhang, Z.-Y. Bai, H. Zhang, W. Lin, L. Wang, and J. Li, J. Lightwave Technol. 32, 1682 (2014).

5. D. Liu, A. K. Mallik, J. Yuan, C. Yu, G. Farrell, Y. Semenova, and Q. Wu, Opt. Lett. 40, 4166 (2015).

6. G. Salceda-Delgado, D. Monzon-Hernandez, A. Martinez-Rios, G. A. Cardenas-Sevilla, and J. Villatoro, Opt. Lett. 37, 1974 (2012).

7. Z. Xu, Q. Sun, B. Li, Y. Luo, W. Lu, D. Liu, P. P. Shum, and L. Zhang, Opt. Express 23, 6662 (2015).

8. V. Bhatia, and A. M. Vengsarkar, Opt. Lett. 21, 692 (1996).

9. H. J. Patrick, A. D. Kersey, and F. Bucholtz, J. Lightwave Technol. 16, 1606 (1998).

10. P. Polynkin, A. Polynkin, N. Peyghambarian, and M. Mansuripur, Opt. Lett. 30, 1273 (2005).

11. Y. Wang, D. N. Wang, M. W. Yang, W. Hong, and P. X. Lu, Opt. Lett. 34, 3328 (2009).

12. Y. Lai, K. Zhou, L. Zhang, and I. Bennion, Opt. Lett. 31, 2559 (2006).

13. G.C.B. Lee, C. Mou, K. Zhou, and K. Sugden, J. Lightwave Technol. 33 , 2561 (2015).

14. J. Zhou, Y. Wang, C. Liao, B. Sun, J. He, G. Yin, S. Liu, Z. Li, G. Wang, X. Zhong, and J. Zhao, Sensor. Actuat. B- Chem. 208, 315 (2015).

15. S. Silva, O. Frazão, J. L. Santos, and F. X. Malcata, Sensor. Actuat. BChem. 161, 88 (2012).

16. S. F. O. Silva, Opt. Eng. 47, 054403 (2008).

17. Z. L. Ran, Y. J. Rao, W. J. Liu, X. Liao, and K. S. Chiang, Opt. Express 16, 2252 (2008)

18. Y. Ma, X. G. Qiao, T. Guo, R. H. Wang, J. Zhang, Y. Y. Weng, Q. Z. Rong, M. L. Hu, and Z. Y. Feng, Chin. Opt. Lett. 10 (2012).

19. H. Y. Choi, G. Mudhana, K. S. Park, U. C. Paek, and B. H. Lee, Opt. Express 18, 141 (2010).

20. P. Chen, X. Shu, H. Cao, and K. Sugden, Opt. Lett. 42, 1157 (2017). 


\section{Full References}

1. J. Li, L. P. Sun, S. Gao, Z. Quan, Y. L. Chang, Y. Ran, L. Jin, and B. O. Guan, "Ultrasensitive refractive-index sensors based on rectangular silica microfibers," Opt Lett 36(18), 3593-3595 (2011).

2. L. P. Sun, J. Li, S. Gao, L. Jin, Y. Ran, and B. O. Guan, "Fabrication of elliptic microfibers with $\mathrm{CO} 2$ laser for high-sensitivity refractive index sensing," Opt Lett 39(12), 3531-3534 (2014).

3. X. Fang, C. R. Liao, and D. N. Wang, "Femtosecond laser fabricated fiber Bragg grating in microfiber for refractive index sensing," Opt Lett 35(7), 1007-1009 (2010).

4. S. Gao, W. Zhang, Z.-Y. Bai, H. Zhang, W. Lin, L. Wang, and J. Li, "Microfiber-Enabled In-line Fabry-Perot Interferometer for HighSensitive Force and Refractive Index Sensing," Journal of Lightwave Technology 32(9), 1682-1688 (2014).

5. D. Liu, A. K. Mallik, J. Yuan, C. Yu, G. Farrell, Y. Semenova, and Q. Wu, "High sensitivity refractive index sensor based on a tapered small core single-mode fiber structure," Opt Lett 40(17), 4166-4169 (2015).

6. G. Salceda-Delgado, D. Monzon-Hernandez, A. Martinez-Rios, G. A. Cardenas-Sevilla, and J. Villatoro, "Optical microfiber mode interferometer for temperature-independent refractometric sensing," Opt Lett 37(11), 1974-1976 (2012).

7. Z. Xu, Q. Sun, B. Li, Y. Luo, W. Lu, D. Liu, P. P. Shum, and L. Zhang, "Highly sensitive refractive index sensor based on composite microfiber knots with Vernier effect," Opt Express 23(5), 6662-6672 (2015).

8. V. Bhatia and A. M. Vengsarkar, "Optical fiber long-period grating sensors," Opt Lett 21(9), 692-694 (1996).

9. H. J. Patrick, A. D. Kersey, and F. Bucholtz, "Analysis of the response of long period fiber gratings to external index of refraction," Journal of Lightwave Technology 16(9), 1606-1612 (1998).

10. P. Polynkin, A. Polynkin, N. Peyghambarian, and M. Mansuripur, "Evanescent field-based optical fiber sensing device for measuring the refractive index of liquids in microfluidic channels," Opt Lett 30(11), 1273-1275 (2005).

11. Y. Wang, D. N. Wang, M. W. Yang, W. Hong, and P. X. Lu, "Refractive index sensor based on a microhole in single-mode fiber created by the use of femtosecond laser micromachining," Optics Letters 34(21), 3328-3330 (2009).

12. Y. Lai, K. Zhou, L. Zhang, and I. Bennion, "Microchannels in conventional single-mode fibers," Optics Letters 31(17), 2559-2561 (2006).

13. G. C. B. Lee, C. Mou, K. Zhou, and K. Sugden, "Optimization and Characterization of Femtosecond Laser Inscribed In-Fiber Microchannels for Liquid Sensing," Journal of Lightwave Technology 33(12), 2561-2565 (2015).

14. J. Zhou, Y. Wang, C. Liao, B. Sun, J. He, G. Yin, S. Liu, Z. Li, G. Wang, X. Zhong, and J. Zhao, "Intensity modulated refractive index sensor based on optical fiber Michelson interferometer," Sensors and Actuators B: Chemical 208, 315-319 (2015).

15. S. Silva, O. Frazão, J. L. Santos, and F. X. Malcata, "A reflective optical fiber refractometer based on multimode interference," Sensors and Actuators B: Chemical 161, 88-92 (2012).

16. S.F.O. Silva, "Optical fiber refractometer based on a Fabry-Pérot interferometer," Optical Engineering 47, 054403 (2008).

17. Z.L. Ran, Y. J. Rao, W. J. Liu, X. Liao, and K. S. Chiang, "Lasermicromachined Fabry-Perot optical fiber tip sensor for high-resolution temperature-independent measurement of refractive index," Optics express 16, 2252-2263 (2008).

18. Y. Ma, X. G. Qiao, T. Guo, R. H. Wang, J. Zhang, Y. Y. Weng, Q. Z. Rong, M.L. Hu, and Z.Y. Feng, "Temperature-independent refractive index measurement based on Fabry-Perot fiber tip sensor modulated by Fresnel reflection," Chin Opt Lett 10(2012).

19. H.Y. Choi, G. Mudhana, K. S. Park, U. C. Paek, and B. H. Lee, "Cross-talk free and ultra-compact fiber optic sensor for simultaneous measurement of temperature and refractive index," Optics express 18, 141-149 (2010).

20. P. Chen, X. Shu, H. Cao, and K. Sugden, "Ultra-sensitive refractive index sensor based on an extremely simple femtosecond-laser-induced structure," Optics Letters 42(6), 1157 (2017). 